\title{
Implementing co-production in traditional statutory mental health services
}

\author{
Michael Norton
}

Citation

Norton M (2018) Implementing co-production in traditional statutory mental health services. Mental Health Practice. doi: 10.7748/mhp.2019.e1304

Peer review

This article has been subject to double-blind peer review and has been checked for plagiarism using automated software Correspondence

nortonmichael92@gmail.com

\section{Conflict of interest}

None declared

Accepted

28 June 2018

Published online

January 2019

\section{Abstract}

Since the publication of A Vision for Change (Department of Health and Children 2006), which sets out the direction for mental health services in Ireland, new approaches to interacting with people who have lived experience of mental ill health have emerged. Co-production is one such approach. Co-production is a word used to describe the creation of a dialogical space where the service user, family members, carers and service providers enter a collaborative medical partnership to improve their own care and also service provision. Co-production is a cornerstone in the delivery of a recovery-oriented service and when implemented correctly it has the power to achieve positive change. The aim of this article is to provide background information and guidance on how to implement co-production in traditional mental health services. It is in line with the publication and implementation of the Irish recovery guidance document, A National Framework for Recovery in Mental Health (Health Service Executive 2017a).

\section{Author details}

Michael Norton, peer support worker and doctoral student, department of nursing and health care, school of health sciences, Waterford Institute of technology, Waterford, Ireland

\section{Keywords}

mental health, mental health service users, management, service development, service redesign

This article has been developed to support the Irish guidance document, A National Framework For Recovery in Mental Health (Health Service Executive (HSE) 2017a). This article spans two years, 2018-2020, with the aim of creating a recovery-oriented service. The framework defines the core principles of recovery and outlines what mental health services need to do to support service users in their recovery. It recognises and values the input of traditional professionals, such as psychiatrists and psychiatric nurses, and of other stakeholders, such as service users, family members or carers and those involved in voluntary sector organisations relating to mental health. The framework covers the following four recovery principles:

» The centrality of the service user's lived experience.

» The co-production of recovery promoting services between all stakeholders. 
》 An organisational commitment to the development of recovery-oriented mental health services.

» Supporting recovery-oriented learning and recovery-oriented practice across all stakeholder groups.

Implementing the second recovery principle, the co-production of recovery promoting services between all stakeholders, is the focus of this article. Co-production is imperative for the creation of recovery services because it recognises the value of learned and experiential knowledge. This is important as those who possess experiential knowledge can increase the understanding of other stakeholders of what recovery means to them and how it can be achieved. It is through experiential knowledge that learning about how recovery is individualised and unique comes about. In this way stakeholders can learn that, due to the individualistic nature of recovery, services need to adapt from their medicalised past to a future that is inclusive of the lived experience. This article sets out a two-year plan that aims to run in conjunction with the national framework for recovery (HSE 2017a).

\section{Context}

Mental health services in Ireland have traditionally been biomedically based, following the structure of UK services (Scott et al 2003). This was evident through past publications such as The Lunacy Regulation (Ireland) Act 1871, by which services followed UK legislation when caring for people deemed clinically insane. Until more recently, mental health services in Ireland still operated through traditional asylums, where service users were often stigmatised and condemned to a lifetime of misery due to their mental status.

In 1984, the Department of Health (DH) published a document: The Psychiatric Services - Planning for the Future (DH 1984), which was aimed at revolutionising mental health services. It identified the value of other supports in the treatment of mental disorders, the contributions from allied health professionals such as social workers and occupational therapists, while identifying the need to make services more community based. However, for more than three decades after the publication of this document, biomedical parameters were still seen as vital to the treatment of mental disorders. This was evident through the publication of the Mental Health Act (Ireland) 2001, which gave power to psychiatrists to incarcerate and coercively treat those who had mental health issues.

More recently the Department of Health and Children (DHC) published a national policy document for Irish mental health services, A Vision for Change (DHC 2006). It was published to implement major reform in the mental health services within a ten-year period. It identified a framework for creating and building positive mental health while helping to provide easier access to services for inpatient and community-based service users. It was the first policy document in Ireland to recommend service user and carer involvement in services (Mental Health Commission 2009). The document brought about the closure of all psychiatric institutions and made services more community based through the creation of nine community healthcare organisations and the development of community multidisciplinary teams. It also discussed the creation of a more person-centred, recovery-oriented service. This policy document is the Irish version of the UK paper, A National Service Framework for Mental Health (DH 1999), which sets out a plan to improve community care in the UK while preventing hospitalisations for those with mental health difficulties. With the publication of A Vision for Change (DHC 2006) and the backing of evidence-based, peer-reviewed literature, personal recovery became realised as a valid option for those who were often viewed as incurable or chronically disabled; services were re-designed so the holistic needs of the service user were central to the treatment process (Powell and Davies 2001, McNulty and Ferlie 2002).

However, Irish services did not begin to incorporate personal recovery into treatment regimens until the establishment of a pilot programme known as Advancing Recovery in Ireland in 2013 (HSE and Advancing Recovery in Ireland 2016), which took inspiration from the UK recovery programme, Implementing Recovery through Organisational Change. This (which????) programme was aimed at making services more recovery-oriented through initiating the ten steps of organisational change, of which one of one was coproduction.

The importance of user involvement activities in aiding recovery has been highlighted with specific attention to how these initiatives change the therapeutic relationship from the traditional doctor-patient relationship to one focused on a co-productive medical partnership (Owens and Cribb 2012). As Figure 1 suggests, there are many degrees of user involvement, from non-participation to tokenism to citizen control (Arnstein 1969). 


\section{Figure 1. A ladder of citizen participation}

However, despite these advances in user participation, Dunston et al (2009) acknowledge that there is a need to have a better process of engagement in health between stakeholders. This level of engagement can only be achieved in an environment in which psychological and social approaches to illness complement that of biomedical parameters (Owens and Cribb 2012).

Co-production, therefore, has emerged as a concept that helps in the delivery of a recovery-oriented service in the mental health arena (Bovaird 2007, Dunston et al 2009).

User participation initiatives such as co-production have been highlighted as one of three interconnecting strands needed for a cultural change in the therapeutic relationship to occur (Dunston et al 2009). However, Owens and Cribb (2012) state that much of the conflict relating to co-production is due to a failure to view health in a more complex manner than taking simply a biomedical approach.

Due to the success of Advancing Recovery in Ireland, the national framework for recovery in mental health (HSE 2017a) was published for Irish services to continue to grow towards recovery through the implementation of principles such as co-production, an aim that inspired the writing of this article.

\section{Defining co-production}

Co-production is a critique of the artificial divide between professionals and other stakeholders (Boyle and Harris 2009a or just 2009???). However, there seems to be a lack of consensus on its definition (Hatzidimitriadou et al 2012, Brandsen and Honingh 2015 , Filipe et al 2017). It is a concept that has different definitions depending on which narrative, culture or discipline is embraced (Pestoff 2012, Cepiku and Giordano 2014). Filipe et al (2017) suggest that this confusion about the definition of co-production could be due to two reasons:

》 Different fields of work could have differing visions on what co-production as a process is.

" Co-production definitions could differ depending on what is being produced, how, by whom and for what purpose.

However, according to Hyde and Davies (2004) and Palumbo (2016), co-production has been identified as a complex, multifaceted process that in its most basic form relies on the input of service users (Ostrom and Ostrom 1977). Service user input is, therefore, at the foundation of the concept (Palumbo 2016). Table 1 identifies in more detail some of the important points about co-production as a concept.

\section{Table 1. The concept of co-production}

\begin{tabular}{|c|c|}
\hline Co-production is... & Explanation \\
\hline Power sharing & The relocation of power from provider to service users. The recognition of the power of the lived experience \\
\hline Creation of an exploratory space & Different perspectives come together to create new knowledge \\
\hline Access to knowledge & To make informed decisions. To have a shared delivery of services \\
\hline A collaborative process & All types of knowledge come together to reach sociably desirable and acceptable outcomes \\
\hline A holistic approach & Clinically acquired knowledge forms only part of a person's recovery \\
\hline A voluntary relationship & A relationship based on consent where all people are equals \\
\hline Non-linear & A relationship that has many obstacles in its way \\
\hline A continuum of practice & Co-production should incorporate every aspect of clinical practice \\
\hline
\end{tabular}

Co-production relies on the premise that service users and providers share a common belief of what is valuable in treatment interventions and overall outcomes (Owens and Cribb 2012). Therefore, co-production affects the interaction between all stakeholders (Palumbo 2016). Co-production is intrinsically linked to all aspects of service provision (Hyde and Davies 2004). This is highlighted by Dunston et al (2009), who identify that the difference between co-production and other initiatives is that it emphasises and relies on partnership practice between all stakeholders (Figures 2 and 3). 


\section{Figure 2. The 'insider' domain of health service production: a co-production view Figure 3. Stakeholders involved in the co-productive process}

The idea behind co-productive practice is to combine professional knowledge with experiential knowledge to strive towards an optimal outcome (Palumbo 2016, Vaeggemose et al 2018). Co-production is a method by which service users become effective members of the multidisciplinary team through the process of using the first person narrative (Palumbo 2016). Co-production can also describe the process of producing better service quality and efficiency outcomes by changing the relationship from one of service dependency to one of service-user control (Ewert and Evers 2014, Scottish Borders Community Planning Partnership 2017).

However, for the purposes of this article, the definition of co-production used will come from the Irish document, A National Framework for Recovery in Mental Health (HSE 2017a), where the New Economics Foundation's definition is used. This states that co-production is where public services are delivered using all perspectives, of professionals, service users and family members, equally and reciprocally (New Economics Foundation 2009 or Boyle and Harris??).

\section{Types of co-production}

According to Loffler (2009), Barker (2010) and Cepiku and Giordano (2014), there are four types of co-production:

» Individualised co-production - appears in services that benefit the active participant and occurs individually, for example, with service users working with peer support workers.

》 Collective co-production - occurs in groups with different stakeholders, for example, Alcoholics Anonymous.

» Additive co-production - service-user input into professional treatment, for example, care planning.

»Substitutive co-production - local governmental input is replaced by input from service users and communities, for example, in consumer panels or local forums.

\section{History of co-production}

Co-production originated from the work of Elinor Ostrom and her research team at the University of Indiana in the 1970s (Ostrom 1996, Cepiku and Giordano 2014, Carr et al 2016). This concept was developed by Edgar Cahn (Boyle et al 2006a, Rethink Mental Illness 2015).

In the 1980s, the concept was introduced to the UK by Anne Coote (Realpe and Wallace 2010) but it did not flourish until the 1990s, when service providers highlighted that there was a need for an alternative model in service delivery (Needham and Carr 2009). According to Boyle et al (2006b), it emerged as a result of the lack of recognition between providers of the successful input of consumers in the delivery of services.

In Ireland co-production is now being introduced as a valuable decision-making process and treatment option in mental health services through the continuous growth of personal recovery and its acknowledgement in A National Framework for Recovery in Mental Health (HSE 2017a). It is now used in all aspects of service provision. It is used, for example, in the preparation, delivery and audit or evaluation of recovery educational programmes such as the Eolas project, designed specifically for service users and family members of those who have a diagnosis of a bipolar or schizophrenia-spectrum disorder. Ireland now has a growing number of recovery colleges embedded into the service to provide recovery educational modules to all stakeholders. Recovery colleges, such as Roscommon-East Galway Advancing Recovery in Ireland and the Recovery College South East, are becoming commonplace in Irish mental health services - and at the heart of these institutions is co-production. It embeds all aspects of the recovery colleges' ethos and practice.

In the delivery of services, Ireland has a number of initiatives to aid all perspectives in this task, most notably in care planning, where the service user is at the heart of all care decisions. This has been further noted in areas such as West Cork, where initiatives such as open dialogue are used to aid in the delivery of services. In the evaluation of services, all perspectives become equal and valid in discussions through local area forums, which came about through the creation of the Mental Health Engagement Office. The Mental Health Engagement Office is an office that was set up by the Irish statutory mental health services, the purpose of which was to develop structure, systems and mechanisms that engaged service users and family members or carers in services (HSE 2017b). It has 
been operational since 2016 and is responsible for the development and growth of both the local and area forums. These forums form the vessels by which such involvement and co-production can occur.

\section{Process of co-production - what is involved?}

Co-production is not easy to implement into the mental health services (Palumbo 2016). However, when fully embraced by the services, co-production can be used in every therapeutic interaction, even in crisis (Alakeson et al 2013). The co-productive process, according to Dunston et al (2009), Realpe and Wallace (2010) and Batalden et al (2016), takes many forms:

》 Co-design and co-governance - service-user involvement in service planning or design, for example, area leads.

» Co-implementation - service-user involvement in the delivery of services, for example, peer support workers.

»Co-evaluation - service-user involvement in service audit or evaluation, for example, peer researchers and consumer academics.

The process varies on the contributors understanding of participation, co-production itself, hierarchy and power dynamics (Filipe et al 2017). However, co-production occurs in three ways (Figure 4):

》 Co-production between, for example, care planning.

》 Co-production between and with, for example, facilitating in recovery colleges.

»Co-production at an organisational level, for example, involvement in initiatives such as Advancing Recovery in Ireland.

\section{Figure 4. Methods of co-production}

The practical outcomes associated with co-production are affected by individual and organisational factors such as motivation (van Eijk and Steen 2014), service readiness (Marlett et al 2015) and resources (Gill et al 2011).

\section{Role of co-production in traditional mental health services}

There are two understandings of co-production that influence its role: a recognition-based or choice-based approach. In the recognition-based approach providers identify the value of the service user's input and transform the services to incorporate them. The choice-based approach gives service users autonomy and self-determination without any previously observed benefits to service provision (Glynos and Speed 2012).

The aim of co-production is to redefine how services are delivered (Munoz et al 2014). This, according to the National Development Team for Inclusion (2016), is achieved through attaining equality for those involved in the therapeutic relationship through the development of an equal partnership between the public sector and citizens (Bettencourt et al 2002, Nikcevic and Jatta 2014, Rethink Mental Illness 2015).

\section{Principles of co-production}

For co-production to become a reality in the mental health services, organisations and individuals need to follow a set of clearly defined principles (Table 2).

\section{Table 2. Principles of co-production}

\section{Co-production principle}

Asset-based model

Active citizenship

Building on the person's capabilities

Mutual responsibility

Breaking down barriers

Facilitating > delivering

\section{Explanation}

Service users become active stakeholders in service provision. Experiential knowledge is seen as valuable and service user-based outcomes are the focus

The transformation of service users to equal partners in service provision

Focuses on the person's strengths rather than their deficits

Stakeholders view each other as equal partners who share responsibility and accountability

Boundaries need to be pushed and borders should be pulled down

Providers should facilitate rather than deliver recovery to service users 
Public services as a catalyst of change rather than the sole provider of services

Mutuality and reciprocity

Flexibility

Peer support - building social networks

Valuing work differently - redefining work
Service providers should incorporate a holistic approach and understand that it is acceptable that they do not always have the answers for service users

Service providers need to interact with service users on a mutual basis where both parties give and take from the relationship

Providers should use an approach that can be adapted to the external environment

Service users should help each other in their recovery journey by sharing their experiences freely to gain or give support

Work should be redefined from a chore with a monetary gain to an activity by which one can develop socially and be included in society

There are several principles that are more difficult to implement into Irish mental health services than others. These include:

》 Active citizenship and mutual responsibility - services will find this more difficult to implement due to the hierarchical system present in health organisations, the power that has been assigned to professional roles and a reluctance to share power and responsibility and to identify service users as equals.

» Building on the person's capabilities - professionals are forced due to the biomedical culture that is ingrained into Irish health services to view a person's deficits rather than their strengths, which they can use in their recovery journey.

» Facilitating - traditional biomedical accounts of health view people with mental health problems as helpless victims of disease who cannot control their recovery. Co-production views this differently as it identifies professionals as facilitators of the user's recovery. However, this could be difficult to achieve due to the biomedical parameters that are present in Irish health services.

\section{Co-productive models: the theory behind practice}

Like its definition, there is no accepted model of co-production (Rethink Mental Illness 2015, National Development Team for Inclusion 2016). This could become problematic in an Irish context as there is no set model to follow in the implementation of coproduction. However, this could also be advantageous as it gives the Irish service providers more room and flexibility to develop what co-production means to them. For the purposes of this article two main theories on co-production as it relates to health will be analysed:

》 Co-production as a propositional theory (Cepiku and Giordano 2014).

» The Governance International (2017) model of co-production.

\section{Co-production as a propositional theory}

This theory highlights the integration of co-production into normal management and planning systems and demonstrates the need for subsequent integration of parallel productions rather than a top-down decision-making process.

Figure 5 summarises this theory based on a number of propositions. The main constructs are represented in the form of coloured boxes. The arrows represent the testable propositions to explain the relationship between constructs. Indirect relationships are indicated by broken lines.

The figure highlights that co-production is a complex process that involves the relationship between service user and provider and encapsulates a holistic approach that allows for the involvement of third parties.

\section{Figure 5. Co-production as a propositional theory}

\section{Governance International model of co-production}

This model demonstrates how user involvement can occur in services. It is used to map co-production that takes place to improve approaches and identify avenues for further exploration. Figure 6 demonstrates that, to integrate co-production in service commissioning and delivery, the following 'co' words need to be present:

» Co-commissioning - management of work alongside other stakeholders in the prioritisation of service planning. 
》 Co-design - providers work with stakeholders to redesign services to produce more efficient outcomes.

» Co-delivery - service users work alongside providers in delivering services.

》 Co-assessment - providers work with communities in the evaluation and audit of services.

\section{Figure 6. Governance International model of co-production}

(Governance International 2017)

The five phases to co-production shown in Figure 6 are the five steps in the co-production journey as highlighted by the Scottish Borders Community Planning Partnership (2017).

» Map it - identify what is already being co-produced and look into where co-production could be introduced.

» Focus it - identify where an impact can be made or where co-production can be valued.

》 People it - recruit people who genuinely want to be involved.

» Market it - advertise co-production initiatives and give incentives to start and maintain them.

》 Grow it - showcase results and promote co-production throughout the services.

According to Grundy et al (2017), however, it is still unclear which components of the different co-production models provide value to the service.

\section{Benefits of co-production}

The advantages of co-production have been recognised as transformative and substantial, particularly to the service user (Dunston et al 2009). The lack of consensus on the definition of co-production has been identified by Stirling (2012) as advantageous as it allows room for the expansion and flexibility of the model.

Co-production benefits the system as it generates profound systemic change (Cahn and Gray 2004). According to Owens and Cribb (2012), this is because co-production allows the provider to view health through a first-person lens, while acknowledging their thirdperson perspective. Therefore, there is no dominant narrative in co-production (Ewert and Evers 2014). This is important because through this unique perspective we can holistically understand the nature of health and identify treatment options that are most suitable for the person.

Dunston et al (2009) and Palumbo (2016) both note that co-production benefits services because it challenges the biomedical model. Part of this is the concept's ability to remove the hierarchy in health to manufacture a dialogical space whereby each participant is seen as equal (Ramirez 1999, Rethink Mental Illness 2015). It is in this way that the concept challenges the idea that staff are 'experts' (Barker 2010). Co-production liberates the service user from the traditional 'dependency culture' created by statutory bodies (Boyle and Harris 2009a without the a??) by providing freedom of choice (Palumbo 2016).

Co-production also benefits services by increasing service quality and helping to reduce costs (Boyle and Harris 2009b without the b??, Palumbo 2016), despite the initial cost necessary to set up co-production (Nikcevic and Jatta 2014).

Service users also benefit from co-production because it improves the care they receive, decreases risk of complications, improves biopsychosocial outcomes and reduces the chance of institutionalisation (Cepiku and Giordano 2014, Palumbo 2016, Scottish Borders Community Planning Partnership 2017). Service users also benefit from this initiative because it increases their social capital (Bovaird and Loeffler 2012, Hatzidimitriadou et al 2012, Nikcevic and Jatta 2014). Co-production also helps service users by increasing their subjective well-being (Slay and Stephens 2013, Nikcevic and Jatta 2014, Palumbo 2016).

Other benefits identified by Dunston et al (2009) include a change in the therapeutic interaction, which reshapes the roles and rules associated with traditional services. This change allows the service user to become partners in service delivery (Palumbo 2016), creating an opportunity for genuine involvement and empowerment (Rethink Mental Illness 2015).

Co-production takes the focus away from psychopathology to a more person-centred process (Boyle et al 2010). As a result the process is more likely to meet the needs of service users (Barker 2010). Therefore, this approach focuses on rebuilding the sense of identity lost through illness by learning new skills, increasing confidence (Hatzidimitriadou et al 2012), improving social relationships, increasing self-worth, improving levels of resilience and quality of life (Slay and Stephens 2013, Nikcevic and Jatta 2014, Nikcevic and Jatta 2015a). This works in conjunction with changing staff attitudes towards users to reduce the number of consultations and hospitalisation rates (Boyle and Harris 2009b without the b??, Slay and Stephens 2013, Nikcevic and Jatta 2015b). 
Co-production has also been shown to increase the likelihood of future volunteerism and involvement in local communities (Hatzidimitriadou et al 2012, Nikcevic and Jatta 2014), and give stakeholders better access to information, which increases compliance (O’Dwyer and Kinsley 2012, Nikcevic and Jatta 2015a). It has also been shown to increase service users' self-esteem and sense of belonging, which, when combined with the development of old and new skills, can improve their chances of gaining employment (Hatzidimitriadou et al 2012, O’Dwyer and Kinsley 2012, Slay and Stephens 2013).

Other benefits of co-production include greater efficiency in the services and higher satisfaction rates (Alford and O'Flynn 2012). These benefits, as highlighted by Durham College and the Regional Municipality of Durham Social Services Department (2016), the HSE (2017 a or b??) and Mayer and McKenzie (2017), are due to the fact that co-production implementation increases service-user control and reduces in stigma. There is also increased acknowledgement of the fact that lived experience is a valued knowledge base in the therapeutic relationship. Co-production benefits stakeholders because it increases service-user empowerment while allowing a cultural shift in professional attitudes, which creates an environment of mutual learning (Hatzidimitriadou et al 2012).

\section{Barriers to co-production}

Co-production has several challenges (Vaeggemose et al 2018) and, according to Cepiku and Giordano (2014) and Phillips and Morgan (2014), incurs significant costs. This is contradicted by Barker (2010), Durham College and the Regional Municipality of Durham Social Services Department (2016) and Filipe et al (2017), who identify that it is of economic benefit to services. This is especially true in rural communities where it may be difficult and expensive to provide public services (Munoz et al 2014).

The biomedical culture that is ingrained in mental health services is a major barrier because staff who are trained in this culture prioritise paternalism, coercion and accountability in their practice, which counteracts co-production values (Newman 2001, Social Care Institute for Excellence et al 2013, National Development Team for Inclusion 2016, Vaeggemose et al 2018). Practices enacting the standards of the biomedical model also decrease the effectiveness of co-production (Alakeson et al 2013). Staff attitudes towards the co-productive process along with the tokenistic involvement of service users, resulting from such negative attitudes, are identified as sub-barriers to co-production. As a result of such tokenistic practices, service users in this relationship often feel more like research subjects rather than true vessels of change. Providers are also hesitant and in some cases reluctant to surrender their power (Hatzidimitriadou et al 2012, Durham College and the Regional Municipality of Durham Social Services Department 2016, Mayer and McKenzie 2017). This lack of willingness to disseminate power leads to a lack of staff participation in co-production (Nikcevic and Jatta 2015b). In the biomedical culture, service policies such as A Vision for Change (DHC 2006) do not translate into practice (Realpe et al 2015). The pressure on staff to conform to cultural and institutional norms also inhibits the development of a more recovery-oriented service (Carr et al 2016).

Co-production is linked to empowerment. This can cause an ideological conflict to occur because mental health systems are based on a hierarchical model that places an emphasis on withholding power (Bhaskar and Danermark 2006). However, Owens and Cribb (2012) emphasise that the challenges to co-production are not all centred around power and autonomy. Carr et al (2016), the National Development Team for Inclusion (2016) and Vaeggemose et al (2018) identify that restrictive professional practice, administrative and legal issues are barriers because they prevent services from creating this cultural change. Adams et al (2014) identify that coproduction requires services to develop more slowly. This would be identified as a barrier because mental health services are fastpaced environments (Whitehouse et al 2013, Nikcevic and Jatta 2014).

Another prominent barrier is that of stigmatisation, especially due to externalised stigma (Nikcevic and Jatta 2014, Vaeggemose et al 2018). This is stigma received from external stimuli such as friends, family, communities and society. Due to this barrier, services often have to rely on the same pool of participants when co-producing (Shortall 2008). This is unsurprising because, to co-produce in the community, one must be identifiable as either a service user or provider. When looking at the evidence it is clear that older participants are less likely to participate in co-productive activities due to the stigma associated with having a mental health difficulty (Barker 2010).

Ethics is another barrier to co-production (Tuurnas et al 2015). This barrier can also be observed in many psychiatric and general departments in the health sector. The main question here concerns consent and whether someone has capacity to make the decision to 
enter into a co-productive relationship. This barrier is intrinsically linked to the barriers that occur due to the presence of the biomedical model. The lack of resources available to those in the co-productive relationship is also a barrier and these include:

》 Lack of relevant information - there is a lack of guidance resources available to help those who wish to create a co-productive service (Tuurnas et al 2015).

》Service location - this can also be considered as a lack of resources, because there may be no viable locations to facilitate coproduction. This can also relate to a lack of participants due to the location of services (Durham College and the Regional Municipality of Durham Social Services Department 2016).

" Lack of participation - this could be due to service users not receiving a monetary gain for their work (Hatzidimitriadou et al 2012), a lack of provider interest in the initiative (Tuurnas et al 2015, Nikcevic and Jatta 2015b, Carr et al 2016), lack of buy in from senior management (O’Dwyer and Kinsley 2012) or a resistance from providers to change (Carr et al 2016, National Development Team for Inclusion 2016). Ultimately this can also be due to a lack of co-operation between stakeholders (Nikcevic and Jatta 2015a).

Another barrier to co-production lies in the professionals themselves (Isett and Miranda 2015). This may be due to differing opinions between third- and first-person narratives (Tuurnas et al 2015). Other barriers include a lack of clear accountability (Cepiku and Giordano 2014), service politics (Nikcevic and Jatta 2014), tokenism (O’Dwyer and Kinsley 2012), as well as the misconception that co-production is not suitable in all contexts (Nikcevic and Jatta 2015a) and that service users know things that professionals do not (Slay and Robinson 2011). Other barriers, according to the literature, include the lack of a definition of what co-production is (Nikcevic and Jatta 2014) along with a lack of necessary skills to implement co-production, and unrealistic expectations from stakeholders on what co-production can achieve (Hatzidimitriadou et al 2012).

Co-production has barriers due to the fact that the priorities of the health systems do not match the service user's societal values (Vaeggemose et al 2018) and, therefore, it is difficult to implement in a health setting (Palumbo 2016).

\section{How to implement co-production}

Co-production underpins a recovery-oriented service (HSE 2018). This document was created to support the national framework for recovery (HSE 2017a) in making co-production a reality, and there are certain actions that must be carried out for co-production to become part of everyday practice (Table 3 ).

\section{Table 3. Making co-production a reality}

\section{Overall action}

Service users define their own recovery goals for their lives using service supports as appropriate. The articulation of selfdetermination is a central component of the care planning process

\section{Measure}

There is evidence in the case notes that interactions with the service support the attainment of recovery goals defined in the care plan

\section{Task}

》 Language is important in all aspects of the service. Using language that is easily understood by all stakeholders helps these individuals to interact and comply more with the service. For the service user recovery goals to be taken into consideration and developed, it is recommended that the language used in all services should be changed

"Staff should be given time and encouraged to develop a close relationship with other stakeholders to view and document their health through a lens that uses all levels of knowledge at the person's disposal

"Staff should be encouraged to create a space where parties come together in equal partnership and have open and honest communication pathways with each other. Staff should also be given training in co-production as it relates to their service

»Professionals should receive training to show that they facilitate service users in their recovery

»Providers should encourage service users to take control of their lives by giving them autonomy and personal responsibility, for example, through service user involvement in care plans

»Service users should be empowered to realise what recovery means to them. This can be achieved through recovery education programmes

The service has or will develop a strategic and policy approach to coproduction, shared decision
The service will record in its service and operational plans the mechanism by which they will coproduce all strategic and policy
》 Power bodies should support a co-production initiative through documentation in service and operational plans

"Service and operational plans should represent a stratified account of health

»Policies should be updated to support co-production while allowing for constant 
making and recovery-

promoting relationships

Services will have capacitybuilding measures on coproduction and opportunities for all stakeholders to participate in co-production

\section{The contribution of all} stakeholders is recognised as having a value attached and the stakeholder is rewarded appropriately

All substantive decisionmaking includes coproduction as part of the decision-making process

The service users', family members' and carers' experience of engaging in mental health services is used to support the design and delivery of services

The service has a strategic approach to engaging lived experience as part of the workforce

The service will adopt an organisational approach to co-produced evaluation strategies to monitor the effectiveness of its recovery approach and will include appropriate validated outcomes and process measures from all stakeholder perspectives

The service has training and practice supports for recovery orientation and practice of staff

documents

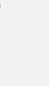

The services can provide data on co-production training and on coproduction in practice

There is an agreed mechanism to remunerate, pay or otherwise value all stakeholders partaking in coproduction

A service user experienced in coproduction to help develop all governance structures of the service

The service has a process to capture on an annual basis a spread of experiences of engaging with the service

The service will begin to introduce lived experience supports into the workforce

The service will measure personal recovery outcomes for service users and families with appropriate tools annually supervision

»Policies and plans should document the need and intention to change the traditional culture

》Mission statements should be redefined

》 The service will have policies and guidelines on how it will engage in co-production

»Staff should complete mandatory training in co-production. This can be achieved through gaining extra continuous professional development points by attending Advancing Recovery in Ireland learning sets. Training should include facilitation skills and open dialogue to realise the value of the lived experience

" An analysis of community resources should be carried out

》Staff should receive protective time to engage in co-production training

»Measures should be brought in so that service users or carers are reimbursed for their time and work without fear of losing their benefits. This should be advertised when recruiting participants for co-production

» The culture should change from a top-down system to a bottom-up approach, by which service users and family members have a say at all levels of the service

" There should be a direct link between service users and other stakeholders through employment of area leads

》 Co-production will allow service users the dialogical space needed so this exchanging relationship can have value, for example, local forums at which service users and family members play an essential part in service provision
» There should be the employment of those with lived experience into recovery-oriented roles for example as peer educators, peer support workers and area leads

» The prevention and elimination of tokenistic practices is paramount and should be made a priority

»Ethical issues should be considered before implementing co-production

»An evaluation tool should be designed and used to evaluate co-productive practices on an annual basis
All staff should have at least two team reflective practice sessions on recovery annually
» Conflicts should be examined using a critical realism approach

»Professionals should identify their personal perceptions of their profession to identify if it affects their practice

"Professionals should reflect on their practice. This is the first step in the co-production process

»Service providers should be given protective time to prepare and deliver co-produced workshops

\section{Conclusion}

Co-production is a multifaceted concept that is difficult to define within a single theoretical framework. Co-production is not only about the sharing of power; it is the realisation that the lived experience itself is a valuable source. It must be carefully used in the health services to minimise the risk of tokenistic practices. However, it is imperative for the future of recovery-oriented practice that co-production is ingrained into health culture and practice. This article was aimed at helping service providers reach this objective while minimising the risk of tokenism. Co-production, when implemented correctly, has the power to achieve positive and lasting change. This article was also aimed at disclosing how to implement co-production into mental health services. It outlines what should be done within a two-year time frame, 2018-2020, for services to become more co-productive and recovery oriented. It is envisioned by the author that in the future there will be an evaluation tool created at the end of the two years, 2020-2021, to identify if services have been co-produced as suggested. 


\section{References}

Adams V, Burke N, Whitmarsh I (2014) Slow research: thoughts for a movement in global health. Medical Anthropology. 33, 3, $179-197$.

Alakeson V, Bunnin A, Miller C (2013) Co-production of Health and Wellbeing Outcomes: The New Paradigm for Effective Health and Social Care. Office for Public Management, London.

Alford J, O’Flynn J (2012) Rethinking Public Service Delivery. Palgrave Macmillan, Basingstoke.

Arnstein S (1969) A ladder of citizen participation. Journal of the American Planning Association. 35, 4, $216-224$.

Barker A (2010) Co-Production of Local Public Services. Local Authorities and Research Councils Initiatives.

www.govint.org/fileadmin/user_upload/publications/LARCI_CoproductionPapers_2010.pdf (Last accessed: 10 January 2019.)

Batalden M, Batalden P, Margolis P et al (2016) Co-production of healthcare services. BMJ Quality and Safety. 7, 509-517.

Bettencourt L, Ostrom A, Brown S et al (2002) Client co-production in knowledge-intensive business services. California Management Review. 44, 4, $100-128$.

Bhaskar R, Danermark B (2006) Metatheory, interdisciplinarity and disability research: a critical realist perspective. Scandinavian Journal of Disability Research. 8, 4, 278-297.

Bovaird T (2007) Beyond engagement and participation: user and community co-production of public services. Public Administration Review. 67, 5, 846-860.

Bovaird T, Loeffler E (2012) We're All in This Together: Harnessing User and Community Co-Production of Public Outcomes. University of Birmingham/Institute of Local Government Studies, Birmingham.

Boyle D, Clarke S, Burns S (2006a) Aspects of Co-Production: The Implications for Work, Health and Volunteering. New Economic Foundation, London.

Boyle D, Clarke S, Burns S (2006b) Hidden Work: Co-production by People outside Paid Employment. Joseph Rowntree Foundation.

www.jff.org.uk/sites/default/files/jrf/migrated/files/9781859354674.pdf (Last accessed: 7 December 2018.)

Boyle D, Harris M (2009a) The Challenge of Co-Production. New Economics Foundation, London.

Boyle D, Harris M (2009b) The Challenge of Co-Production. www.assetbasedconsulting.co.uk/uploads/publications/The_Challenge_of_Co-production.pdf (Last accessed: 7 December 2018.) (Is this the same as Boyle and Harris 2009a??)

Boyle D, Slay J, and Stephens L (2010) Public Services Inside Out: Putting Co-production into Practice. New Economic Foundation, London. https://media.nesta.org.uk/documents/public services inside out.pdf (Last accessed: 10 January 2019.)

Brandsen T, Honingh M (2015) Distinguishing different types of co-production: a conceptual analysis based on the classical definitions. Public Administration Review. 6 , $3,426-435$

Cahn E, Gray C (2004) Reciprocal Co-Production. Working Colloquium Series. Bloomington IA. (Please check this ref?? Who's the publisher? ? Is it in Indiana (IN)? rather than lowa (IA)???)

Carr S, Coldham T, Roberts A et al (2016) Position Paper: Are Mainstream Mental Health Services Ready to Progress Transformative Co-Production? National Development Team for Inclusion, London.

Cepiku D, Giordano F (2014) Co-production in developing countries: insights from the community health workers experience. Public Management Review. 16, 3, 317340 .

Department of Health (1984) The Psychiatric Services - Planning for the Future: Report of a Study Group on the Development of the Psychiatric Services. www.lenus.ie/hse/handle/10147/45556 (Last accessed: 7 December 2018.)

Department of Health (1999) A National Service Framework for Mental Health. London, The Stationery Office. www.dh.gov.uk/en/Publicationsandstatistics/Publications/PublicationsPolicyAndGuidance/DH_4009598 (Last accessed: 21 January 2019.)

Department of Health and Children (2006) A Vision for Change: Report of the Expert Group on Mental Health Policy. The Stationery Office, Dublin.

Dunston R, Lee A, Boud D et al (2009) Co-production and health system reform: from re-imagining to re-making. Australian Journal of Public Administration. 68, 1, 3952 .

Durham College, Regional Municipality of Durham Social Services Department (2016) Co-Production: A Literature Review and Environmental Scan. Durham College, Durham.

Ewert B, Evers A (2014) An ambiguous concept: on the meaning of co-production for health care users and user organisations? International Journal of Voluntary and Nonprofit Organizations. 25, 2, 425-442. 
Filipe A, Renedo A, Marston C (2017) The co-production of what? Knowledge, values, and social relations in health care. PLOS Biology. 15, 5, e2001403-e2001406.

Gill L, White L, Cameron I (2011) Service co-creation in community-based aged healthcare. Managing Service Quality. 21, 2, 152-177.

Glynos J, Speed E (2012) Varieties of co-production in public services: time banks in a UK health policy context. Critical Policy Studies. 6, 4, 402-433.

Governance International (2017) Co-production. www.govint.org/our-services/co-production (Last accessed: 7 December 2018.)

Grundy AC, Walker L, Meade $\mathrm{O}$ et al (2017) Evaluation of a co-delivered training package for community mental health professionals on service user- and carerinvolved care planning. Journal of Psychiatric and Mental Health Nursing. 24, 6, 358-366.

Hatzidimitriadou E, Mantovani N, Keating F (2012) Evaluation of Co-Production Processes in a Community-Based Mental Health Project in Wandsworth. Kingston University/St. George's University of London, London.

Health Service Executive (2017a) A National Framework for Recovery in Mental Health: A National Framework for Mental Health Service Providers to Support the Delivery of a Quality Person-Centred Service. Advancing Recovery in Ireland, Dublin.

Health Service Executive (2017b) The Mental Health Engagement Office. www.hse.ie/eng/services/list/4/mental-health-services/mentalhealthengagement/apply (Last accessed: 10 January 2019.)

Health Service Executive (2018) Recovery Education Guidance Paper 2018-2020. Advancing Recovery in Ireland, Dublin.

Health Service Executive, Advancing Recovery in Ireland (2016) Advancing Recovery Ireland: A Guidance Paper on Implementing Organisational and Cultural Change in Mental Health Services in Ireland. http://hdl.handle.net/10147/613321 (Last accessed: 11 January 2019.)

Hyde P, Davies H (2004) Services design, culture and performance: collusion and co-production in health care. Human Relations. 57, 11, 1407-1426.

Isett K, Miranda J (2015) Watching sausage being made: lessons learned from the co-production of governance in a behavioural health system. Public Management Review. 17, 1, 35-56.

Loffler E (2009) Why Co-Production Is an Important Topic for Local Government. www.govint.org/fileadmin/user upload/publications/coproduction why it is important.pdf (Last accessed: 10 January 2019.)

Marlett N, Shklarov S, Marshall D et al (2015) Building new roles and relationships in research: a model of patient engagement research. Quality of Life Research. 24, $5,1057-1067$

Mayer C, McKenzie K (2017) '...It shows that there's no limits': the psychological impact of co-production for experts by experience working in youth mental health. Health and Social Care in the Community. 25, 3, 1181-1189.

McNulty T, Ferlie E (2002) Re-Engineering Healthcare: The Complexities of Organisational Transformation. Oxford University Press, Oxford.

Mental Health Commission (2009) From Vision to Action: An Analysis of the Implementation of a Vision for Change. MHC, Dublin

Munoz S, Farmer J, Warburton J et al (2014) Involving rural older people in service co-production: is there an untapped pool of potential participants? Journal of Rural Studies. 34, 212-222.

National Development Team for Inclusion (2016) Practical Guide: Progressing Transformative Co-Production in Mental Health. www.ndti.org.uk/uploads/files/MH_Coproduction_guide.pdf (Last accessed: 7 December 2018.)

Needham C, Carr S (2009) Co-production: An Emerging Evidence Base for Adult Social Care Transformation. www.scie.org.uk/publications/briefings/briefing31 (Last accessed: 7 December 2018.)

New Economics Foundation (2009) The Challenge of Co-Production. www.neweconomics.org/2009/12/challenge-co-production (Last accessed: 7 December 2018.) (Is this the same as Boyle and Harris 2009a??)

Newman J (2001) Modernising Governance: New Labour, Policy and Society. Sage, London.

Nikcevic A, Jatta F (2014) Evaluation of Co-production in Mental Health (Phase 1). www.rethink.org/media/1658117/phase 1 evaluation report.pdf (Last accessed: 7 December 2018.

Nikcevic A, Jatta F (2015a) Evaluation of Co-production in Mental Health (Phase 2). http://www.rethink.org/media/1543634/Phase\%202\%20Evaluation\%20Report.pdf (Last accessed: 10 January 2019.)

Nikcevic A, Jatta F (2015b) Evaluation of Co-production in Mental Health (Phase 2, Five-Month Follow-Up).

www.rethink.org/media/1658122/evaluation_of_coproduction_in_mental_health_phase_2_-_5_month_follow_up_27_october_2015.pdf (Last accessed: 11 January 2019.) 
O'Dwyer J, Kinsley G (2012) Service User Involvement - Staff Training Manual. www.srdatf.ie/wp-content/uploads/2015/06/FINAL_Service-User-Manual-March2015.pdf (Last accessed7 December 2018.)

Ostrom E (1996) Crossing the great divide: co-production, synergy and development. World Development. 24, 6, 1073-1087.

Ostrom V, Ostrom E (1977) Public goods and public choices. In Savas E (Ed) Alternatives for Delivering Public Services: Towards Improved Performance. Westview Press, Boulder CO.

Owens J, Cribb A (2012) Conflict in medical co-production: can a stratified conception of health help? Journal of Health Philosophy and Policy. 20, 3, 268-280.

Palumbo R (2016) Contextualizing co-production of health care: a systematic literature review. International Journal of Public Sector Management. 29, 1, 72-90.

Pestoff V (2012) Co-production and third sector social services in Europe: some concepts and evidence. International Journal of Voluntary and Non-profit Organizations. 23, 4, 1102-1118.

Phillips A, Morgan G (2014) Co-production within health and social care: the implications for Wales. Quality in Ageing and Older Adults. 15, 1, 10-20.

Powell A, Davies H (2001) Business process re-engineering: lost hope or learning opportunity? British Journal of Healthcare Management. 7, 11, 446-449.

Ramirez R (1999) Value co-production: intellectual origins and implications for practice and research. Strategic Management Journal. 20, 1, 49-65.

Realpe A, Wallace L (2010) What Is Co-Production? www.qi.elft.nhs.uk/wp-content/uploads/2017/01/what_is_co-production.pdf (Last accessed: 7 December 2018.)

Realpe A, Wallace L, Adams A et al (2015) The development of a prototype measure of the co-production of health in routine consultations for people with long-term conditions. Patient Education and Counseling. 98, 12, 1608-1616.

Rethink Mental IIIness (2015) Co-Production in Commissioning. Getting Started. www.rethink.org/media/1658112/getting_started_guide.pdf (Last accessed: 7 December 2018.)

Scott T, Mannion R, Marshall M et al (2003) Does organisational culture influence health care performance? A review of the evidence. Journal of Health Services Research and Policy. 8, 2, 105-117.

Scottish Borders Community Planning Partnership (2017) An Introduction to Co-Production. www.scotborders.gov.uk/downloads/file/2226/an_introduction_to_coproduction (Last accessed: 7 December 2018.)

Shortall S (2008) Are rural development programmes socially inclusive? Social inclusion, civic engagement, participation and social capital: exploring the differences. Journal of Rural Studies. $24,4,450-457$.

Slay J, Robinson B (2011) In This Together. Building Knowledge about Co-Production. www.cnwl.nhs.uk/wp-content/uploads/2013/01/In_This_Together.pdf (Last accessed: 7 December 2018.)

Slay J, Stephens L (2013) Co-Production in Mental Health: A Literature Review. https://b.3cdn.net/nefoundation/ca0975b7cd88125c3e ywm6bp311.pdf (Last accessed: 10 January 2019.)

Social Care Institute for Excellence (2013) Co-Production in Social Care: What It Is and How to Do It. www.ndti.org.uk/uploads/files/Coproduction_Guide51Fin.pdf (Last accessed: 10 January 2019.)

Stirling A (2012) Opening up the politics of knowledge and power in bioscience. PLoS Biology. 10, 1, e1001233-e1001235.

Tuurnas S, Stenvall J, Rannisto P et al (2015) Coordinating co-production in complex network settings. European Journal of Social Work. 18, 3, 370-382.

Vaeggemose U, Ankersen P, Aagaard $\mathrm{J}$ et al (2018) Co-production of community mental health services: organising the interplay between public services and civil society in Denmark. Health and Social Care in the Community. 26, 1, 122-130.

van Eijk C, Steen T (2014) Why people co-produce: analysing citizens' perceptions on co-planning engagement in health care services. Pubic Management Review. 16, $3,358-382$

Whitehouse S, Lam P, Balka E et al (2013) Co-creation with TickiT: designing and evaluating a clinical ehealth platform for youth. JMIR Research Protocols. 2, 2, e42. 